\title{
Original Research \\ Utilization, Benefits, and Impact of an e-Consultation Service Across Diverse Specialties and Primary Care Providers
}

\author{
Erin Keely, MD, FRCPC, ${ }^{1-3}$ Clare Liddy, MD, MSc, CCFP, FCFP, ${ }^{4,5}$ \\ and Amir Afkham, BEng ${ }^{6}$ \\ ${ }^{7}$ Division of Endocrinology/Metabolism, The Ottawa Hospital, \\ Ottawa, Ontario, Canada. \\ Departments of ${ }^{2}$ Medicine and ${ }^{4}$ Family Medicine, University of \\ Ottawa, Ottawa, Ontario, Canada. \\ ${ }^{3}$ Ottawa Hospital Research Institute, Ottawa, Ontario, Canada. \\ ${ }^{5}$ CT Lamont Primary Healthcare Research Centre, Bruyère \\ Research Institute, Ottawa, Ontario, Canada. \\ ${ }^{6}$ The Champlain Local Health Integration Network, Ottawa, \\ Ontario, Canada.
}

\begin{abstract}
Background: Access to specialist advice remains a barrier for primary care providers (PCPS) and their patients. Virtual consultations have been used to expedite access. There are few studies demonstrating the utilization and impact of such services. We established a regional e-consultation service that was used across a wide range of specialty services and PCPs. Materials and Methods: We prospectively collected all e-consultations submitted from April 1, 2011 to June 30, 2012. Utilization data collected included number of econsultations submitted, specialist response, and time required for the specialist to complete the e-consultation. Perceived benefit to the PCPs and their patients and the impact on care delivery were determined from a close-out survey. Results: Fifty-nine PCPs submitted 406 e-consultations to 16 specialty services. The specialist provided an answer without requesting further information in 89\% of cases, with $>90 \%$ of cases taking $<15$ min for the specialist to complete. Seventy-five percent of cases were answered in $<3$ days. The service was perceived as highly beneficial to providers and patients in $>90 \%$ of cases. In 43\% of submitted cases a traditional referral was originally contemplated but was now avoided. Conclusions: We successfully implemented an e-consultation service across diverse PCPs and specialty services that was highly valued. Almost half of referrals submitted would have required a face-to-face consultation if the service had not been available. Thus e-consultation has tremendous potential for improving access to specialist advice in a much more timely manner than the traditional referral-consultation process.
\end{abstract}

Key words: e-health, dermatology, telemedicine

\section{Introduction}

n 2007, 3 million Canadians reported seeing a specialist for a new condition in the preceding year. ${ }^{1}$ The traditional referralconsultation process requires the patient meet with the specialist before recommendations are provided to the primary care provider (PCP). Canada has the second longest wait times for seeing a specialist compared with 10 other countries according to both primary care physicians and their patients. Forty-one percent of patients waited greater than 2 months to see a specialist, and 73\% of primary care physicians identified long waits to see a specialist as a frequent barrier to patient care. ${ }^{2,3}$ The median time between the referral being made and the specialist appointment has increased 156\%, from 3.7 weeks in 1993 to 9.5 weeks in $2011 .^{4}$ There is an urgency to improve access to specialist advice to reduce delays in diagnosis, to institute timely, effective treatments, and to alleviate patient anxiety., ${ }^{5,6}$

Virtual consultations, where there is no direct contact between the specialist and patient, are used to access to specialist advice in a more expedient fashion. These may include telephone consultations between providers (which are often frustrating because of the need for both parties to be available at the same time), real-time or asynchronous telemedicine (which requires access to specialized equipment), and e-mail (which does not meet current privacy requirements for sharing personal health information). ${ }^{7}$ The limitations of these consultation models have led to the development of Web-based electronic consultations that allow information to be exchanged between providers in a secure environment. There have been few studies demonstrating the utilization and impact of such services. Those that are reported are generally limited to one specialty service ${ }^{8-11}$ or used in healthcare settings where PCPs and specialists share an electronic health record facilitating access to the patient's history and results. ${ }^{12-}$

${ }^{14}$ There is no Canadian experience reported in the literature.

Through the Champlain BASE (Building Access to Specialists through e-consultation) project we established a secure, Web-based e-consultation regional service that was used across a wide range of specialty services and diverse primary care practices. We describe the utilization, the perceived benefits to providers and their patients, and the impact on need for face-to-face consultations of this unique e-consultation service.

\section{Materials and Methods}

\section{DEVELOPMENT OF THE E-CONSULTATION SERVICE}

The development of the Champlain BASE service was previously described. ${ }^{15}$ In brief, the system was built on an existing, 


\section{KEELY ET AL.}

secure Web-based "Regional Collaboration Space" that was sponsored and established by the Local Health Integration Network and hosted within the secure infrastructure of one of the community hospitals. The Collaboration Space is built using standard off-the-shelf platforms and components, and it incorporates several levels of physical and logical security. A comprehensive Threat Risk Assessment had been successfully completed for this environment, and a standard Privacy Impact Assessment was performed for the e-consultation application specifically.

Access to the Regional Collaboration Space is controlled through several different measures. In addition to the standard robust firewall infrastructure, an additional gateway enforces granular access controls and policies. The e-consultation service has its own secure site within the collaboration space. Each user must have a unique username and complex password, and each user must be explicitly added to specific permission groups that grant him or her the necessary visibility and capabilities associated with the user's assigned role. There are four distinct user roles:

1. The PCP, and up to three designated delegates, can have visibility of all cases initiated by that individual PCP.

2. The specialist has visibility of all cases responded to by that individual specialist.

3. The assigner has visibility of PCP name and specialty selected by the PCP for cases pending assignment.

4. The administrator has full access to the system to provide support and troubleshoot if needed.

PCPs submit a patient-specific clinical question to a specialty service, by using a standardized electronic form. Supplementary patient information, such as laboratory results, digital images, and health history, can be attached. The assigner role is responsible for assigning the e-consult to the appropriate specialist. The specialist receives a notification via e-mail (which includes a link to the secure Web site) that there is an e-consult waiting for his or her review and response. For each consultation, depending on the request and information provided, the specialist can (a) provide recommendations and avoid the need for a face-to-face consultation, (b) request additional information before being able to provide advice, or (c) recommend a formal referral, in which case any additional diagnostic tests or courses for treatment could be suggested and initiated before the appointment.

Both the specialist and the PCP receive e-mail notifications at each stage of the process when they know the status of the e-consultation and can log back into the system to see the response and/or provide more information as needed. Iterative communication may occur when either the specialist or PCP asks for clarification or additional information. In addition, a permanent record of the e-consultation is created, which may be downloaded into the patient's health record.

Upon completion and before the case can be officially closed, the PCP completes a mandatory four-question close-out survey with optional free text fields. Two questions on the value of the e-consultation to the PCP and the perceived value to the patient in the specific case submitted are answered using a 5-point Likert scale. The other two questions assess the impact of the advice received on the PCP's course of action and on the need for a face-to face consultation. Respondents are encouraged to include free text comments. Specialists self-report the time required to complete the e-consultation and are paid a prorated hourly rate based on this time. PCPs are not remunerated.

\section{SETTING AND PARTICIPANTS}

This study reports on the results of the pilot phase, which took place from April 1, 2011 to June 30, 2012. The study took place in the Champlain region of Eastern Ontario-an area of 17,600 square miles, with a population of 1.2 million. There is one tertiary-care hospital with three campuses that provides many of the specialty services to people living throughout the region.

PCPs were self-identified after learning about the service through presentations or word of mouth. Specialty services were added based on feedback from the primary care participants and interest expressed from specialists. The great majority are fee-for-service, not salaried, clinicians. PCPs selected which referrals were made through the e-consultation service and which were made through traditional routes.

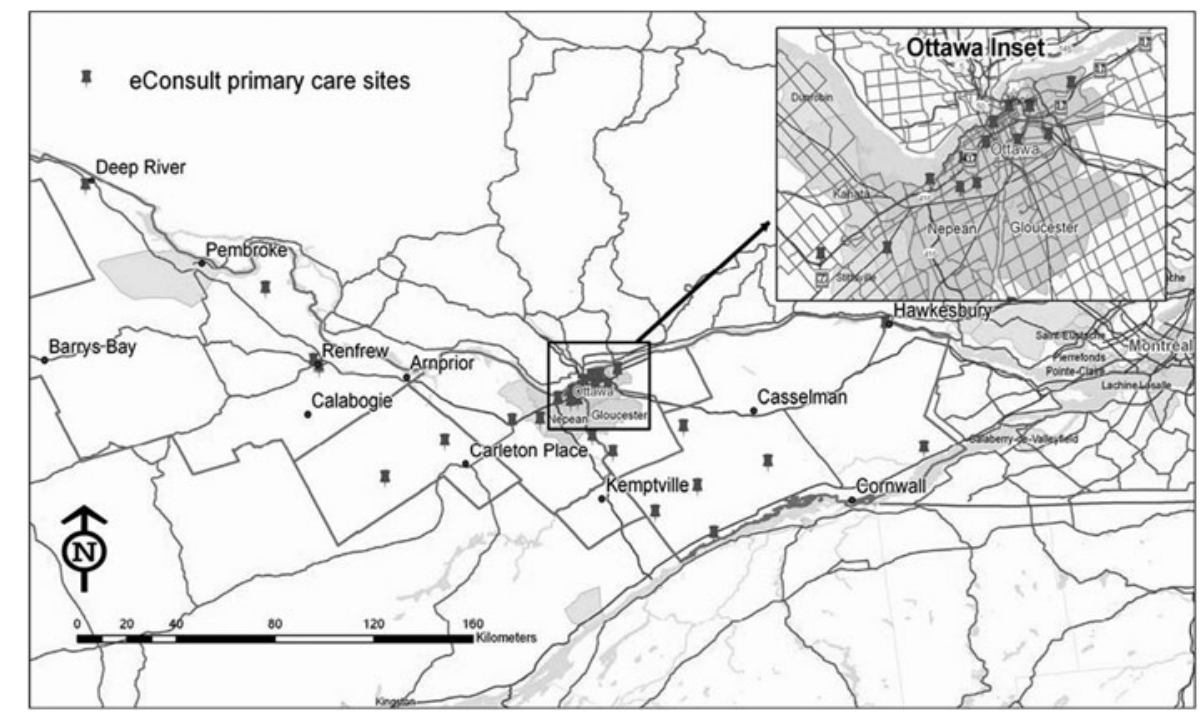

Fig. 1. Distribution of primary care practices that participated in the e-consultation service. 


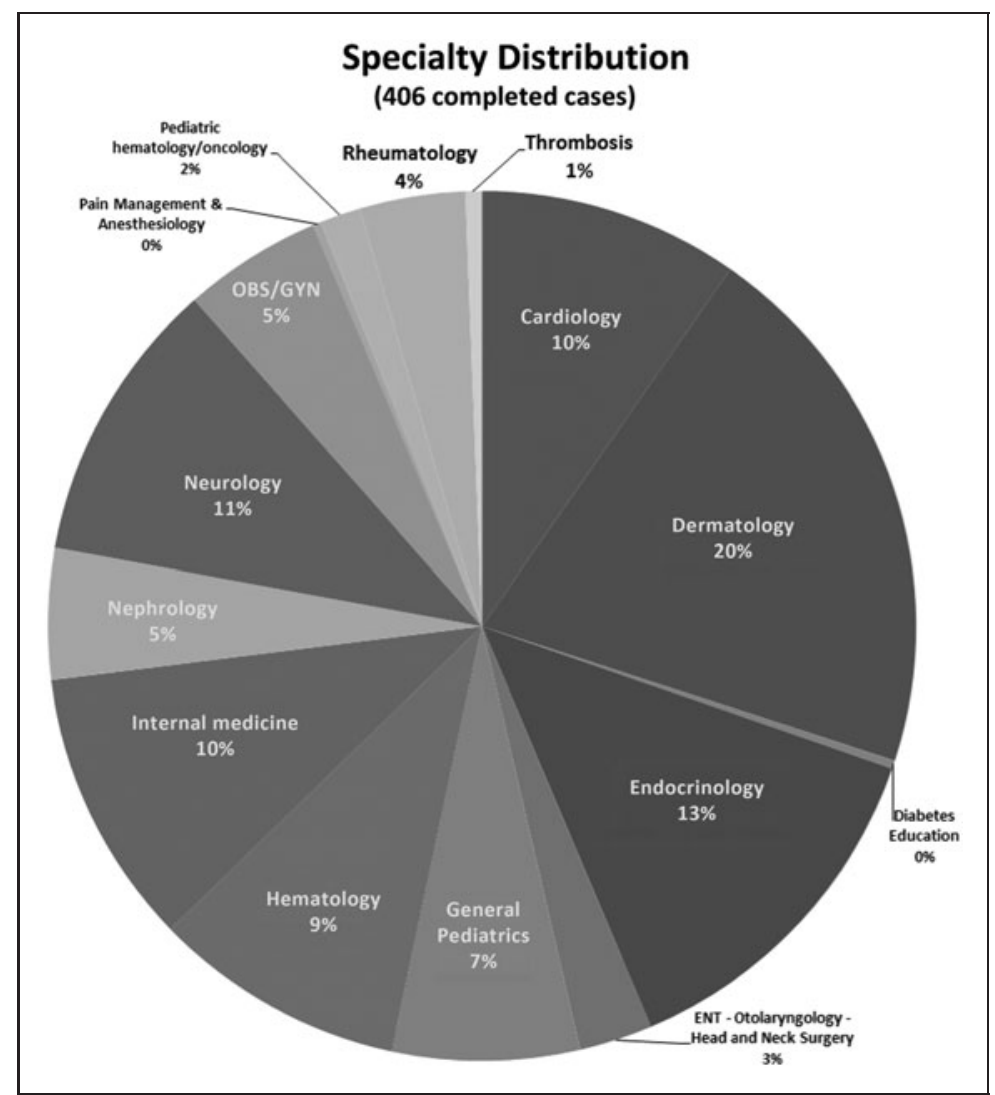

Fig. 2. Distribution of e-consultations by specialty service. ENT, ear, nose, and throat; OBS/GYN, obstetrics/gynecology.

\section{DATA SOURCES}

All e-consultations submitted and close-out surveys were prospectively collected and coded. Utilization data included number of e-consultations submitted per PCP, number of e-consultations directed to each specialty service, specialist response, and time required for the specialist to complete the e-consultation. Perceived benefit to the PCPs and their patients and impact on care delivery were determined from the closeout survey. Survey results were compiled for all individual specialty services with $>10$ completed e-consultations.

Ethics approval was received from the Ottawa Hospital Research Ethics Board.

\section{Results}

Fifty-nine PCPs (48 MDs, 11 nurse practitioners) of the 122 registered submitted at least one e-consultation (median of five). The PCPs participating were located in both rural and urban areas throughout the region (Fig. 1). There were, in total, 406 e-consultations directed to the 16 specialty services. The specialty services receiving the most e-consultations were dermatology (20\%), endocrinology (13\%), neurology (11\%), internal medicine (10\%), cardiology (10\%) and hematology (9\%) (Fig. 2).

The specialist provided an answer without requesting further information in 89\% of the cases, requested more information in $1.5 \%$, and suggested a face-to-face consultation in $9 \%$. The self-reported time it took for the specialist to complete the econsultation was < $10 \mathrm{~min}$ in 57\% of cases, $10-15 \mathrm{~min}$ in 34\%,

\section{Table 1. Examples of Comments Provided by Primary Care Providers on the Benefits of the e-Consultation Service}

\section{SPECIALTY}

SERVICE

\begin{tabular}{|c|c|}
\hline \multirow[t]{2}{*}{ Cardiology } & "I liked the detail in the response which reviewed some basics that I will be able to apply other patients." \\
\hline & "Saved me and the patient a lot of time. Quick easy answer for me-but information that I didn't know or have quick access to." \\
\hline \multirow[t]{3}{*}{ Dermatology } & "Very quick and detailed response-extremely useful-this patient was on a 12 month wait list for dermatology." \\
\hline & "Good review of possible diagnosis and treatment options, very reassuring for the patient." \\
\hline & "Very fast response. Avoided referral to a dermatologist and travel to another town for an elderly patient." \\
\hline Endocrinology & "Such a great way to get an answer to a smallish question that I would worry about but doesn't need any formal consult. Thanks!!" \\
\hline General Pediatrics & "Excellent advice-able not to send someone on to a consultation." \\
\hline \multirow[t]{2}{*}{ Hematology } & "I will be able to provide more information to the consultant, for a more effective visit." \\
\hline & "How exciting to be able to get an answer so quickly and efficiently." \\
\hline \multirow[t]{2}{*}{ Internal Medicine } & "This is a very useful 'corridor consult' venue for the community based doc." \\
\hline & "Reassuring on a course of action taken with extra action suggested." \\
\hline Nephrology & "Although the eConsult wasn't helpful, it at least let me know that I wasn't wasting resources or patient time by making the referral." \\
\hline Obstetrics/Gynecology & "Great additional advice/teaching, I believe this eConsult will improve patient outcomes." \\
\hline Rheumatology & "Very timely response was given and it was helpful knowing I was on the right track without having to wait months to get into the \\
\hline
\end{tabular}

\section{COMMENT}




\section{KEELY ET AL.}

15-20 min in 9\%, and over 20 min in $1 \%$. The time from the e-consultation being initiated by the PCP to the initial response by the specialist was a median of $19 \mathrm{~h}$, with 75\% of e-consultations answered within 2.7 days. The PCP closed the e-consultation (i.e., received and read response and answered close-out survey) within a median time of 5.3 days, with 75\% fully completed within 11.2 days.

The close-out survey results showed that the e-consultation service was highly beneficial to providers and patients. The PCPs felt that the overall value of the e-consultation service in the submitted case was excellent or very good in 95\% of cases. The PCPs perceived the value of the e-consultation service to the patient as excellent or very good in 92\% of cases. Examples of

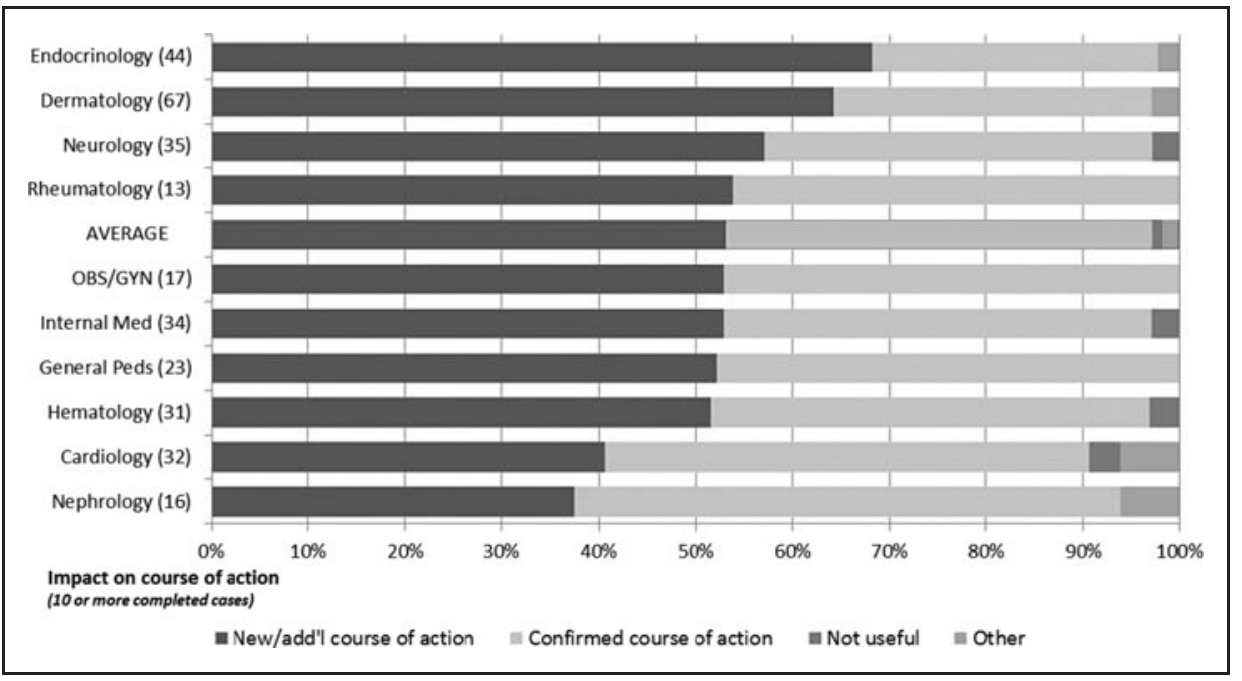

Fig. 3. Impact of e-consultation on the course of action by the primary care provider by specialty service $(n=406)$. OBS/GYN, obstetrics/gynecology; Peds, pediatrics. open-ended comments regarding the ben-

\section{efits of the e-consultation service are given in Table 1.}

The impact of the e-consultation service on course of action of the PCP and need for face-to-face consultations are shown by specialty service in Figures 3 and 4. Overall, 56\% of cases resulted in the PCP getting good advice for a new or additional course of action, and 41\% of cases allowed the PCP to confirm a course of action he or she had in mind. Only $1 \%$ of responses were deemed not to be useful. In $43 \%$ of the cases submitted a referral was originally contemplated but was now avoided. In 29\% of cases a referral was not originally contemplated and was still not needed, but the e-consultation provided useful feedback/information. In 19\% of cases a referral was originally contemplated and was still needed, but the PCP perceived that the e-consultation would lead to a more effective specialist visit. In $3 \%$ of cases a referral was not originally contemplated, but the e-consultation process resulted in a referral being initiated. For the specialty services hematology, endocrinology, and dermatology, over $50 \%$ of the cases would have required a face-to-face consultation if e-consultation was not available.

\section{Discussion}

We successfully implemented an e-consultation service across diverse PCPs and specialty services that was highly valued by participating PCPs. We demonstrated that almost half of re- ferrals submitted to an e-consultation service would have required a face-to-face consultation if the service had not been available. The majority of the e-consultations took $<10 \mathrm{~min}$ for the specialist to answer and were completed in less than 3 days. The potential impact of e-consultations includes not only how many faceto-face consultations can be avoided and reducing wait times, but can also improve communication between primary and specialty care.

There are few reports on the benefits and impact of e-consultation services, none of which occurred within the Canadian healthcare system. ${ }^{16}$ Most studies have been in large healthcare organizations, often

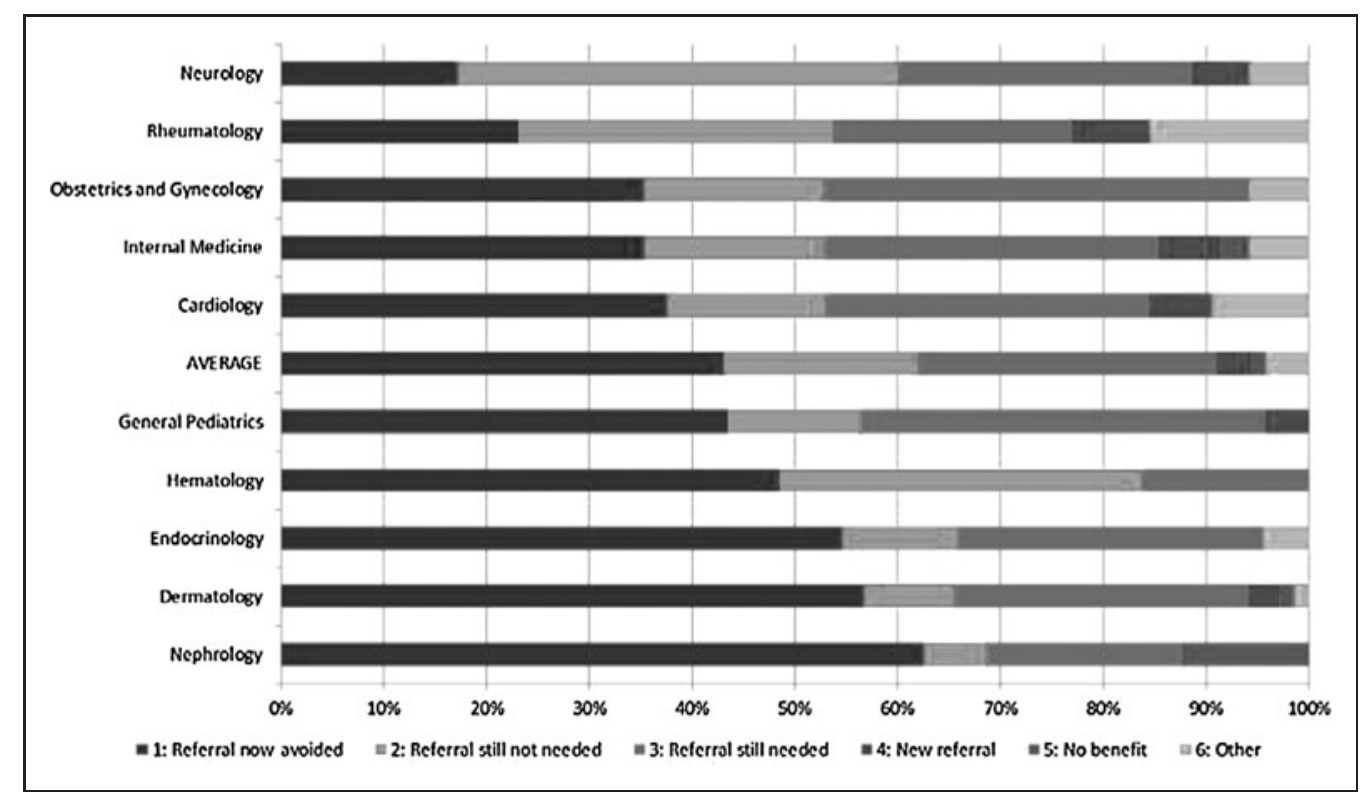

Fig. 4. Impact of e-consultation on need for face-to-face referral by specialty service $(n=406)$. 


\section{IMPACT OF AN E-CONSULTATION SERVICE}

with a shared electronic health record ${ }^{12-14}$ or specialty specific. ${ }^{8-11}$ The group with the most robust evaluation is the Public Safety Net program originating from the San Francisco General Hospital, which was piloted in 2005 and remains active. Primary care physicians and specialists communicate through a referral program embedded in the patients' electronic medical record. A clinician reviews submitted cases to determine if the referral question can be answered through an e-consultation. They have demonstrated shorter wait times in the gastroenterology clinic, reduction in inappropriate referrals being seen in specialist clinic slots, improved clarity of communication between the PCP and specialist, and perceived improvements in overall care. ${ }^{12,17,18}$

However, the authors highlight that access to a shared electronic healthcare record and salaried physicians were critical success factors. Neither of these factors was present in our system. The Doc2Doc system, originating from the University of Oklahoma, was successfully implemented in the Oklahoma Department of Corrections using fee-for-service specialists. Although the study is not yet published, the Principal Investigator reports almost 100,000 e-consultations have taken place and that there has been a 50\% reduction in specialist visits. ${ }^{16}$ Given our satisfaction, utilization, and response times are similar to those reported by others, we are optimistic that these outcomes will be realized with continued utilization and expansion to more PCPs. ${ }^{14,17-20}$ Our study has some limitations, including small sample size, thus limiting our ability to assess impact on wait times. In addition, as we did not collect patient identifiers, we were unable to verify if the patient was seen in a faceto-face consultation following the e-consultation. It is difficult to know if our results are generalizable across primary care and specialist practices. Our PCPs were self-selected, and our specialists within the specialty services were selected by the researchers based on their expressed interest. The percentage of PCPs who submitted questions after being registered on the e-consultation system is similar to the adoption rate at the Mayo Clinic. ${ }^{13}$ It will be important to study the impact across a larger group of providers and in other jurisdictions.

\section{Conclusions}

The Champlain BASE project successfully implemented a highly satisfactory e-consultation service that has the potential to improve access to specialty services through rapid response rates and reduction in the need for face-to-face consultations. E-consultation has tremendous potential for improving access to specialist advice in a much more timely manner than the traditional referral-consultation process.

\section{Acknowledgments}

The Champlain BASE project team included Sean O'Brien, Julie Maranger, Charles Adamson, and Glenn Alexander. We would like to thank the primary care providers and specialists who participated in this pilot project. As well, we would like to thank Glenn Alexander (CIO, Champlain LHIN) for his support and advocacy. Funding was provided by the Ottawa Hospital Academic Medical Organization
Innovation Fund, e-Health Ontario, and the Champlain Local Health Integration Network.

\section{Disclosure Statement}

No competing financial interests exist.

\section{REFERENCES}

1. Carriere G, Sanmartin C. Waiting time for medical specialist consultations in Canada, 2007. In: Health Reports. Catalogue No. 82-003-XPE. Ottawa: Statistics Canada, 2010;21:1-8.

2. Schoen C, Osborn R. The Commonwealth Fund 2010 International Health Policy Survey in eleven countries. Available at www.commonwealthfund.org/ Surveys/2010/Nov/2010-International-Survey.aspx (last accessed November 23, 2012).

3. Schoen C, Osborn R, Squires D, Doty M, Rasmussen P, Pierson R, Applebaum S. A survey of primary care doctors in ten countries show progress in use of health information technology, less in other areas. Health Aff (Millwood) 2012;31:2805-2816.

4. Barua B, Rovere M, Skinner BJ. Waiting your turn. Wait times for healthcare in Canada. 2011 report. 2011. Available at www.fraserinstitute.org/uploadedFiles/ fraser-ca/Content/research-news/research/publications/waiting-your-turn2011.pdf (last accessed November 23, 2012).

5. Bichel A, Erfle S, Wiebe V, Axelrod D, Conly J. Improving patient access to medical services: Preventing the patient from being lost in translation. Healthc O 2009;13(Spec No):61-68.

6. Ontario Medical Association. OMA principles and recommendations: Models and processes of delivery for specialty care. October 2011. 2011. Available at www.oma.org/Resources/Documents/ModelsandProcessesofDeliveryforSpecialty Care.pdf (last accessed August 13, 2012).

7. Caffery $\sqcup$, Smith AC. A literature review of email-based telemedicine. In: Smith AC, Maeder AJ (eds). Global Telehealth. Amsterdam: IOS Press, 2010:20-34.

8. Knol A, van den Akker TW, Damstra RJ, de Haan J. Teledermatology reduces the number of patient referrals to a dermatologist. J Telemed Telecare 2006;12: 75-78.

9. Eminovic N, de Keizer NF, Wyatt JC, Riet G, Peek N, van weert HC, BruijnzeelKoomen CA, Bindels PJ. Teledermatologic consultation and reduction in referrals to dermatologists. A cluster randomized controlled trial. Arch Dermatol 2009;145:558-564.

10. Stoves J, Connolly J, Cheung CK, Grange A, Rhodes P, O'Donoghue D, Wright J. Electronic consultations as an alternative to hospital referral for patients with chronic kidney disease: A novel application for networked electronic health records to improve the accessibility and efficiency of healthcare. Qual Saf Health Care 2010;19:e54.

11. Rushakoff RJ, Woeber KA. Evaluation of a "formal" endocrinology curbside consultation service: Advice by means of the internet, fax, and telephone. Endocr Pract 2003;9:124-127.

12. Kim-Hwang JE, Chen AH, Bell DS, Guzman D, Yee HF, Kushel MB. Evaluating electronic referrals for specialty care at a public hospital. J Gen Intern Med 2010;25:1123-1128.

13. Angstman KB, Robrer JE, Adamson SC, Chaudhry R. Impact of e-consults on return visits of primary care patients. Health Care Manage (Frederick) $2009 ; 28: 253-257$

14. Abbott KC, Mann S, DeWitt D, Sales L, Kennedy S, Poropatich RK. Physician-tophysician consultation via electronic mail: The Walter Reed Army Medical Center Ask a Doc System. Milit Med 2002;167:200-204.

15. Liddy C, Rowan M, Afkham A, Maranger J, Keely E. Building access to specialist care through e-consultation. Open Med 2013;7(1)e1-e8.

16. Horner K, Wagner E, Tufano J. Electronic consultations between primary and specialty care clinicians: Early insights. Issue Brief. Commonwealth Fund. October 2011. 2011. Available at www.commonwealthfund.org/Publications/ 


\section{KEELY ET AL.}

Issue-Briefs/2011/0ct/Electronic-Consultations.aspx (last accessed November 23, 2012).

17. Chen AH, Kushel MB, Grumbach K, Yee HF. Practice profile. A safety-net system gains efficiencies through 'eReferrals' to specialists. Health Aff (Millwood) 2010;29:969-971.

18. Kim Y, Chen AH, Keith E, Yee HF, Kushel MB. Not perfect, but better: Primary care providers' experiences with electronic referrals in a safety net system. J Gen Intern Med 2009;24:614-619.

19. Bergus GR, Sinift SD, Randall CS, Rosenthal DM. Use of an e-mail curbside consultation service by family physicians. J Fam Pract 1998;47:357-360.

20. Bergus GR, Emerson M, Reed DA, Attaluri A. Email consultations: Well formulated clinical referrals reduce the need for clinic consultation. J Telemed Telecare 2006;12:33-38.
Address correspondence to: Erin Keely, MD, FRCPC The Ottawa Hospital, Riverside Campus 1967 Riverside Drive, Room 4-01 Ottawa, ON K1H 7W9

Canada

E-mail: ekeely@toh.on.ca

Received: January 8, 2013

Revised: March 1, 2013

Accepted: March 1, 2013 